Cristina Romaña 1,2

Laure Emperaire 1,2 Ana Maria Jansen 3

\section{Enfoques conceptuales y propuestas metodológicas para el estudio de las interacciones entre el medio ambiente y la salud: aplicación a un programa de investigación sobre la tripanosomiasis americana}

\author{
Conceptual approaches and methodological \\ proposals for the study of interactions between \\ environment and health: application to a research \\ program on American trypanosomiasis
}

1 Institut de Recherche pour le Développement. C.P. 7091, Lago Sul, Brasília, DF 71619-970, Brasil. cromana.necker@invivo.edu emperair@uol.com.br 2 Centro de Desenvolvimento Sustentável, Universidade de Brasília. SAS Quadra 05, Bloco H, 2o andar, Brasília, DF

70070-914, Brasil.

3 Departamento

de Protozoologia, Instituto Oswaldo Cruz, Fundação Oswaldo Cruz. Av. Brasil 4365, Rio de Janeiro, $R J$ 21045-900, Brasil. jansen@gene.dbbm.fiocruz.br

\begin{abstract}
Modifications of the landscape by human activity and migratory movements contribute to the emergence or reemergence of zoonotic and human diseases, particularly those transmitted by insects which often escape the changing environmental conditions, adapting and modifying their trophic networks and morphology, including their genotype. A better understanding of relationships between ecological factors, human factors, and anthropozoonoses is vital to be able to identify variables that allow one to map the risk for human populations. This is the main objective of the research program Landscape Ecology, Land-Use Dynamics, and EcoPathogenic Complexes: Eco-Epidemiologic Risk in the Case of American Trypanosomiasis, developed in various Brazilian ecosystems. The authors describe the program's conceptual and methodological basis and highlight the role of eco-epidemiology for studying the structure and function of natural and anthropogenic foci of infection. Modeling spatial and temporal dynamics can help predict and monitor such tropical diseases.
\end{abstract}

Key words Chagas Disease; Emerging Communicable Diseases; Surveillance; Environment and Public Health

Resumen Las modificaciones del paisaje causadas por las actividades y migraciones humanas contribuyen en hacer emerger o re-emerger enfermedades tropicales, particularmente aquellas cuya transmisión se realiza por insectos vectores que a menudo deben escapar a las condiciones cambiantes ambientales, adaptándose y modificando sus redes tróficas, su morfología, hasta su genotipo. Obtener una mejor comprensión de las relaciones entre los factores ecológicos y humanos y las antropozoonosis es vital para poder identificar variables que permitan cartografiar el riesgo para las poblaciones humanas. Este es el objetivo del programa de investigación Ecología del Paisaje, Dinámica de los Agro-Ecosistemas y Complejos Eco-Patógenos: La Definición del Riesgo Eco-Epidemiológico en la Tripanosomiasis Americana que se desarrolla en diferentes ecosistemas del Brasil. Los autores describen las bases conceptuales y metodológicas del programa e insisten sobre el rol del método eco-epidemiológico para el estudio de la estructura y el funcionamiento de los focos naturales y antropizados de la infección. Modelizar su dinámica espacial y temporal permite concebir nuevos útiles de predicción y de vigilancia.

Palabras clave Enfermedad de Chagas; Enfermedades Transmisibles Emergentes; Vigilancia; Medio Ambiente y Salud Pública 
Los ecosistemas de América Latina, en particular los bosques húmedos tropicales, son actualmente el centro de interés de debates nacionales e internacionales sobre la gestión a largo plazo de la diversidad biológica en el marco de un desarrollo sostenible. En América Central, por ejemplo, la cuenca del Canal de Panamá $\left(3.300 \mathrm{~km}^{2}\right)$ es un extraordinario sistema de producción y de almacenaje del agua dulce que se sitúa en el área metropolitana $\mathrm{Pa}-$ namá/Colón donde se encuentran también los más intensos procesos de urbanización y de industrialización. El gobierno de Panamá hace frente hoy día a un verdadero desafío: el de administrar el canal interoceánico, una de las arterias principales del transporte y del comercio internacional mientras que organiza, alrededor de éste, la protección de sus bosques tropicales húmedos que disminuyen, día a día, bajo la intensa presión de cerca de dos tercios de los habitantes del país (Heckadon-Moreno et al., 1999). En el Brasil, los bosques tropicales húmedos, los cerrados y las caatingas también sufren profundas transformaciones (MMA, 1998; Nepstad et al., 2000). Nuevos proyectos de planeamiento territorial se encuentran ya en curso como por ejemplo el de Avança Brasil (cuenca amazónica) que producirá, sin tardar, transformaciones significativas en los modos de ocupación de las tierras (MT, 2003). El gobierno brasileño prevee así, de aquí al año 2010, aumentar considerablemente las infraestructuras en Amazonía. Entre otros, $6.245 \mathrm{~km}$ de caminos construídos en el bosque serán asfaltados.

Estas dinámicas - controladas o no - llevan al establecimiento de nuevos mosaicos de paisajes donde predominan las formaciones abiertas y fragmentos de la vegetación anterior más o menos alterada y conllevan, muchas veces a largo plazo, graves problemas económicos locales, nacionales y/o regionales en términos de costos para la salud pública. Las actividades humanas, con la disminución de los bosques y la modificación de los nichos ecológicos de los animales y de los insectos vectores de agentes patógenos, se transforman entonces en responsables directa o indirectamente de la disparidad espacial y temporal de endemias como el paludismo, las leishmaniosis o las arbovirosis.

Así, antropisación del paisaje y migraciones humanas contribuyen, en una amplia medida, en hacer emerger o re-emerger enfermedades tropicales humanas o animales, particularmente aquellas cuya transmisión se realiza por insectos vectores (Garrett, 1994; Hoare, 1962; Schatzmayer, 2000; Walker et al., 1996; Wilson et al., 1994). Cualquier modificación del ambiente, que sea debida a un fenómeno natural o a una intervención humana, transforma el contexto ecológico en donde los diferentes elementos que intervienen en el complejo patógeno - huéspedes, vectores y agentes patógenos - cohabitan y circulan desde hace varios millones de años. Las poblaciones de insectos hematófagos (flebótomos, simúlidos, mosquitos o triatominos) son particularmente sensibles a las transformaciones del medio y a las variaciones climáticas y deben escapar a menudo a las condiciones cambiantes ambientales, adaptándose y modificando sus redes tróficas, su morfología, hasta su genotipo. Pueden pasar así de una dieta de tipo zoofílica a una dieta de tipo antropofílica buscando como adaptarse al nuevo ambiente. Estos problemas son particularmente manifiestos en el caso de los triatomíneos (Hemiptera, Triatominae), insectos hematófagos vectores del parásito Trypanosoma (Schyzotrypanum) cruzi, agente responsable de la enfermedad de Chagas o tripanosomiasis americana (Dujardin et al., 1999; Schofield et al., 1999).

Este fenómeno de adaptación dinámica y contínua a las modificaciones del medio ambiente, permite clasificar a los triatominos en especies totalmente silvestres, especies en vías de adaptación - que se encuentran a la vez en ambientes silvestres y domésticos - y especies totalmente adaptadas a las habitaciones humanas (Zeledón \& Rabinovich, 1981). En Brasil, por ejemplo, de las 123 especies de triatominos, 46 se encuentran dispersas en el medio ambiente, 8 se encuentran en vías de adaptación y una sola, Triatoma infestans, inmigrante clandestina de Bolivia que se capturaba únicamente en las casas y dependencias, se encuentra en vías de erradicación gracias a las campañas de control con insecticidas químicos y a la educación sanitaria (Silveira \& Vinhaes, 1999).

En realidad, la enfermedad de Chagas se asemeja a un enorme iceberg cuyo tercio superior emerge como una antropozoonosis y el resto, lo que es la zoonosis, está sumergido en el medio ambiente y poco explorado hasta ahora. Si las campañas de control de la enfermedad de Chagas contra las especies de insectos domiciliadas han sido un éxito en algunos de los 27 países que sufren de esta endemia, es importante, al mismo tiempo, implementar estudios científicos sobre esta zoonosis. La eliminación de especies domiciliadas como T. infestans o Rhodnius prolixus presupone que se redefinan, para un control adecuado de este problema, un cierto número de acciones que impidan - como lo han descrito diversos autores (Dias, 1988, 1991, 2000; FUNASA/MS, 1998; Forattini et al., 1983) - una nueva colonización de 
las habitaciones humanas por otras especies de triatominos. Estas acciones deben ser permanentes mientras se mantenga el riesgo vigente. El riesgo debe ser también identificado, caracterizado y administrado en ausencia de enfermedad y de insectos vectores clásicamente estudiados. La enfermedad de Chagas adquiere hoy día el aspecto de lo que los profesionales de la salud y los de la comunicación llaman una enfermedad emergente - es el caso de la cuenca amazónica (Coura et al., 2002) - y reemergente en zonas ya declaradas indemnes de transmisión por especies clásicamente domiciliadas pero dónde existe el riesgo de nuevas colonizaciones por otras especies, llamadas especies secundarias o candidatos vectores. En estos dos casos, la prevención debe pasar por la construcción de gradientes posibles de riesgo para las poblaciones locales.

Esto significa que deben sustentarse y difundirse nuevas acciones de vigilancia entomológica - ciertos organismos oficiales como en el Estado de São Paulo, en Brasil o en Panamá ya tomaron o están tomando decisiones en este sentido - con una nueva visión del problema que implica una estrategia mejor de lucha contra los vectores de la enfermedad de Chagas. La caracterización técnica del riesgo, en este caso, debe pasar entonces no sólo por la estimación de la importancia relativa de los ciclos silvestres (historia natural de la infección por T. cruzi), las capacidades de dispersión de las especies y su plasticidad genética a la domiciliación (genética y dinámica de las poblaciones) sino también por el estudio de variables culturales (comportamientos y actividades humanas, representaciones del riesgo y de la enfermedad), socio-económicas y políticas (migraciones humanas, desarrollo económico) y epidemiológicas (seropositividad a T. cruzi) de las poblaciones expuestas. Esto supone la necesidad de dar nuevos enfoques conceptuales y metodológicos a los estudios epidemiológicos hasta ahora pertinentes, integrando a los conceptos clásicos provenientes de disciplinas tales como la ecología o la geografía, herramientas útiles como la eco-epidemiología o la geo-informática.

Este es el objetivo del programa franco-brasileño de investigación Ecología del Paisaje, Dinámica de los Agro-Ecosistemas y Complejos Eco-Patógenos: La Definición del Riesgo EcoEpidemiológico en la Tripanosomiasis America$n a$ (EDCTA) en el Brasil (Conselho Nacional de Desenvolvimento Científico e Tecnológico/Fundação Oswaldo Cruz/Institut de Recherche pour le Développment, no 910054/00-6). Se trata, a partir de una reflexión multidisciplinar, de reforzar la importancia de estudios de este tipo en la medida en que el parasitismo es un fenómeno ecológico en el que el medio ambiente ejerce sobre éste presiones selectivas y que consecuentemente las diferencias regionales observadas son frecuentemente atribuídas, de una forma reduccionista, a cualidades intrínsecas del parásito.

\section{La noción de riesgo}

El riesgo en salud pública se define como "todos los acontecimientos indeseables que pueden afectar la salud de los individuos, los grupos o las poblaciones a partir de comportamientos, actividades humanas y situaciones" (CNRS, 1998:8). La aparición reciente de enfermedades como el SIDA o la encefalopatía espongiforme bovina y las consecuencias nocivas de la exposición a agentes químicos tóxicos como el asbesto, el mercurio, el radón o ciertos insecticidas, conformó una reacción contundente y masiva de la opinión pública quién se fue rápidamente sensibilizando al concepto de riesgo. La opinión pública demostró entonces una gran capacidad para movilizarse, exigiendo la intervención de los responsables públicos de los sectores afectados lo que llevó a efectuar rápidamente un diagnóstico de situación y a organizar los estudios científicos y técnicos necesarios que permitieran la caracterización del riesgo y su gestión. Sin embargo, en el caso de las enfermedades tropicales transmitidas por insectos vectores, el diálogo entre las poblaciones expuestas al riesgo y los responsables de las políticas de la salud es casi inexistente.

El objetivo del programa EDCTA consiste, a partir de estudios comparativos en diversos ecosistemas brasileños, en poner de manifiesto las interacciones que pueden haber entre los riesgos epidemiológicos, los modos de ocupación del espacio y el manejo del medio ambiente. De esta forma se supone:

- Obtener una mejor comprensión de las relaciones entre factores ecológicos, factores humanos y antropozoonosis con la finalidad de identificar variables indicadoras de riesgo.

- Desarrollar herramientas metodológicas nuevas en ecología aplicada a la epidemiología. El programa toma sobretodo en consideración el concepto de paisaje como espacio global de transmisión de la enfermedad y de las actividades humanas, integrando así las nociones de complejidad y de multiplicidad de las escalas espaciales y temporales.

- Aplicar los resultados, a corto plazo y localmente, restituyendo los resultados a las pobla- 
ciones locales y efectuando campañas de sensibilización sobre el concepto de riesgo y de vigilancia; a mediano y largo plazo contribuyendo, en una escala nacional o regional, a la creación o a la consolidación de redes de observatorios de investigación sobre medio-ambiente y salud o de observatorios de vigilancia de enfermedades emergentes o re-emergentes.

\section{Enfoques conceptuales}

En este programa, se da la mayor importancia al estudio del medio ambiente y a la dimensión ecológica de los elementos del complejo patógeno tal como Rioux (2001) lo describió en sus trabajos sobre el estudio de las leishmaniosis en Francia y en Marruecos. Ciertos conceptos clásicos deben entonces ser enunciados:

\section{El complejo eco-patógeno}

El conjunto de los organismos que participan en la expresión de una enfermedad en un momento y en un contexto geográfico dado, constituyen un sistema ecológico llamado complejo eco-patógeno (Rioux, 2001; Sorre, 1943). La interdependencia de los elementos bióticos de este complejo confiere al sistema ciertas características en la estructura y en la estabilidad. Las interconexiones existentes son de tipo coadaptativo y en consecuencia son mantenidas por procesos de co-evolución. El funcionamiento del conjunto (con circulación del agente causal de un huésped a otro, animal o humano) depende entonces de factores endógenos y exógenos. Tales sistemas tienen por lo tanto su propia estructura y su propia dinámica. La representación de estos complejos legitimiza entonces el uso de modelos particulares de tipo sistémico (Rioux, 1977).

\section{El paisaje epidemiógeno}

Las poblaciones de parásitos circulan entre poblaciones de insectos vectores y animales silvestres o domésticos, cuyas especies y distribución varían apreciablemente según las localidades consideradas, y/o poblaciones humanas. Estos tres elementos, al ser identificados y su ecología conocida, se deben integrar en un marco general, el de la biocenosis o paisaje epidemiógeno.

En el caso de la tripanosomiasis americana, formulamos la hipótesis que se trata de una zoonosis que se presenta fundamentalmente en la forma de focos de infección (Pavlosky, 1939; Petrischeva, 1971), dejando de lado las formas de transmisión inter-humana, congénita, por transfusión sanguínea y por transplantes de órganos. En cada foco, T. cruzi se integra a los ecosistemas en los cuales evoluciona según circuitos extremadamente precisos, a través de numerosos y diversos huéspedes. La parasitocenosis, representada por la cadena de estos huéspedes, se convierte entonces en un aspecto restringido de esta biocenosis. Desde este punto de vista, las áreas endémicas de la infección son estrechamente dependientes de las biocenosis correspondientes.

\section{Focos de infección y heterogeneidad espacio-temporal}

En el concepto de foco de infección, limitado en el espacio, la enfermedad se concibe como el resultado de factores edáficos, climáticos, de vegetación y flora, y humanos. Mientras estos factores sean constantes, la enfermedad perdura. No obstante, una de las características fundamentales y a menudo no tomada en cuenta del foco de infección, reside en su heterogeneidad espacial y temporal. Es así como, de un lugar a otro, las densidades y el comportamiento de los elementos del complejo pueden variar considerablemente, implicando modificaciones eco-epidemiológicas significativas. El análisis ecológico del área de estudio debe entonces tomar en cuenta las variaciones espaciales de las estructuras del foco, la incertidumbre del muestreo realizado en el conjunto de la zona, pudiendo éste ser reducido si se trabaja en función de una estratificación espacial de las variaciones conjuntas de las densidades de vectores y de las frecuencias de infección humana y/o animal (Rioux et al., 1990).

Los triatominos tienen también características de comportamiento trófico y demográfico que intervienen de una forma radical en la focalización de la infección. Es lo que lleva el nombre de focos “à précellence vectorielle” antropozoonosis transmitida por un vector invertebrado cuyo rol es determinante en el funcionamiento y la dinámica del sistema parasitario (Rioux, 2001; Rioux et al., 1969).

\section{Estrategias de las poblaciones de triatominos}

El programa toma también en cuenta el tipo de dinámica poblacional que tienen los triatominos. Estos obedecen a una estrategia demográfica de tipo $\mathrm{k}$ definida por una necesidad de habitar nichos extremadamente estables, de tener una capacidad biótica limitada (constante k) con índices de natalidad y de dispersión 
relativamente bajos y una esperanza de vida larga del adulto (potencial reproductivo). La regulación de la población se efectúa básicamente a través del índice de natalidad.

La dinámica poblacional también es dependiente del acceso a la alimentación. Se sabe que para un número fijo de huéspedes sobre quienes alimentarse y una alta densidad de triatominos, cada insecto no podrá alimentarse convenientemente porque el huésped reaccionará, importunado por tantas picaduras, interrumpiendo la comida del triatoma. Como consecuencia, ocurrirá un retraso en las mudas (cinco mudas son necesarias para alcanzar el estadio adulto), un aumento en la duración del ciclo vital, una caída de la tasa de oviposición de las hembras, un aumento en la dispersión de adultos que vuelan, un cambio en el ratio del sexo en el favor de los machos y un aumento en la mortalidad. Además su tasa de recombinación genética es muy lenta. Es interesante observar que la estrategia de otras poblaciones de insectos como los mosquitos o los flebótomos, son de tipo r, es decir, altamente oportunistas, colonizando medios poco estables, con altas tasas de natalidad y un tiempo de generación corto, dando así crecimientos de tipo exponencial. Estas consideraciones sobre las dinámicas poblacionales tienen el objeto de encaminar la modelización estadística de los muestreos y la cartografía de las áreas de riesgo.

\section{Muestreos y escalas geográficas}

Los focos de infección no pueden ser estudiados sin tomar en cuenta una serie de situaciones concretas, muy diversas, claramente definidas desde un punto de vista ecológico. Las adquisiciones de datos deben ser realizadas a un mismo nivel espacio-temporal y a una misma escala geográfica. Esta es la razón por la cual el enfoque eco-epidemiológico que se utiliza en el programa EDCTA como metodología de trabajo en el campo, constituye un paso innovador en el estudio de este problema de salud pública. Se trata de asimilar y de restituir, en un conjunto, los resultados de los estudios de campo y de laboratorio de las diferentes disciplinas y se caracteriza por una metodología única general (Rioux et al., 1990). En muchos estudios ya realizados, los indicadores que se evalúan suelen ser índices globales a nivel de un continente (Rogers, 2000). En nuestro caso, las escalas utilizadas son a nivel del foco de trabajo, entre $1 / 25.000$ y $1 / 100.000$.

\section{Enfoques metodológicos}

\section{La ecología aplicada a la epidemiología}

La ecología parece ser la disciplina la más adaptada al análisis de los procesos y de los mecanismos complejos que entran en juego, directa o indirectamente, en los focos "naturales” de infecciones. Según Rioux et al. (1990), el conocimiento del complejo patógeno debe comportar la recolección de un gran número de datos gracias a estudios de campo de tipo ecológico que aportan información a tres niveles diferentes:

- Primer nivel: la historia natural de la infección. Una identificación precisa de todos los organismos que componen el complejo patógeno es necesaria, la mayoría de las veces utilizando datos de archivos y bibliografía especializada. La geografía permite entonces proyectar en mapas, las distribuciones de casos humanos, de huéspedes, de reservorios y de vectores. Se define así, grosso modo, el foco natural de la infección. Se elaboran entonces modelos descriptivos que permiten la jerarquización de los elementos que intervienen en el ciclo, situando e integrando las diferentes disciplinas para establecer las mejores técnicas de enfoque del estudio.

- Segundo nivel: el estudio ecológico de los elementos identificados en el primer nivel. En esta etapa, se estudia la ecología de cada elemento identificado, comprendida la ecología humana. Este tipo de análisis aporta datos cuantitativos y cualitativos sobre los organismos, la dinámica de sus poblaciones, así como datos epidemiológicos sobre la incidencia y la prevalencia de la enfermedad.

Los métodos de estudio en el campo comprenden técnicas de muestreos y una estratificación ecológica del espacio. La estratificación consiste en cortar el espacio en sectores homogéneos con respecto a un factor discriminante (Rioux, 2001). Se trata de una zonificación basada en un elemento "focalizador", es decir, aquel que tiene bajo su dependencia el funcionamiento del ciclo en el foco. Como la mayoría de las veces este elemento es dificilmente identificado, se le reemplaza por un indicador (Rioux, 2001). Entre los indicadores, se pueden citar ejemplos como el clima local, la altitud, la geomorfología, la pedología. Desde 1969, Rioux et al. proponen como indicador la vegetación, que tiene como ventaja integrar los factores abióticos y tener una cierta estabilidad que puede ser transcripta en mapas a diferentes escalas. En el caso del género Rhodnius, por ejemplo, diferentes especies de palmeras del com- 
plejo Attalea constituyen buenos indicadores de su presencia (Romaña et al., 1999). La zonificación del área de estudio sirve fundamentalmente para establecer un transecto-itinerario que atraviese todos los estratos representativos. En cada estrato, se buscan las correlaciones que puedan existir entre los diferentes elementos del complejo y los elementos del medio. Para el estudio del riesgo temporal, se pueden definir diferentes escalas de tiempo que van de las variaciones estacionales a las variaciones pluri-anuales. Los muestreos deben entonces realizarse en las diferentes épocas del año, siguiendo los mismos estratos representativos del área y utilizando las mismas técnicas de muestreo.

- Tercer nivel: el estudio ecológico de la transmisión. Se trata de un estudio sistemático del ciclo que tiende a delimitar las modalidades de las interacciones entre las poblaciones de parásitos y los huéspedes o entre los diferentes huéspedes, para dar un sentido y una intensidad al flujo de circulación del agente patógeno ya que durante su evolución, el parásito seguirá circuitos muy precisos.

El conjunto sistematizado de estos tres niveles conduce a definir el concepto de riesgo epidemiológico en sus tres componentes, espacial, temporal y humano y a definir así estrategias realistas y eficaces de control, de prevención y de vigilancia de enfermedades transmitidas por vectores (De La Rocque et al., 2001; Rioux, 1990).

\section{El rol de la geografía}

Los estudios pioneros de Luiz Jacintho da Silva (1999) en el Estado de São Paulo (Brasil) ya permitieron concebir la importancia de la geografía en el estudio y control de la enfermedad de Chagas. Silva demuestra claramente, basándose también en la teoría de los focos naturales de Pavlovsky, en el concepto de espacio geográfico - o espacio nosológico - y en la noción de endemia chagásica, cómo la caracterización del espacio y su proceso de organización y desarticulación como territorio - espacio organizado como reflejo de una sociedad - pueden ser determinantes para la distribución, la evolución y la interrupción de la transmisión natural de la enfermedad de Chagas. Adoptando una posición similar a la de Forattini (1980) en sus trabajos también pioneros sobre la biogeografía de los triatominos domiciliados, insiste sobre la necesidad de reflexionar en términos de dinamismo, integrando metodológicamente la noción de tiempo con una reconstrucción histórica del espacio nosológico. En el caso del programa EDCTA, la geografía se integra a los objetivos conceptuales y metodológicos en diferentes niveles de reflexión y de trabajo, gracias a técnicas puestas a punto recientemente (De La Rocque et al., 2001). En efecto, la síntesis del conjunto de los factores ecológicos efectuados actualmente por estudios de imágenes por satélite permite, en un principio, la delimitación de estratos para el muestreo. Los sistemas de información geográficos (SIG), por ejemplo, pueden describir los fenómenos estudiados lo que implica la construcción de bancos de datos a partir de variables georeferenciadas. Estas variables se obtienen a diferentes escalas, pudiendo ir de la genética poblacional de los triatominos a la demografía de la población humana local expuesta. Uno de los objetivos del programa reside en llegar a integrar la estructura y la dinámica del foco natural de infección en el que se trabaja, al paisaje global y ayudar, así, a distribuir, en gradientes y a una escala regional, el riesgo epidemiológico (CNRS, 1998). Los análisis matemáticos o estadísticos de estos fenómenos permiten al mismo tiempo pasar de la descripción del foco natural de infección a su interpretación lo que implica la realización de modelos.

La modelización de la dinámica espacial y temporal de los focos de infección que están en constante evolución lleva, en una nueva etapa, a crear útiles de predicción y de vigilancia y a adoptar medidas preventivas. Las interacciones posibles entre los SIG operacionales y los bancos de datos del medio ambiente pueden también ser útiles para evaluar el rol de los cambios climáticos en la emergencia y la reemergencia de las enfermedades infecciosas.

Los aportes metodológicos son esenciales entonces para la organización de las actividades, su seguimiento, la administración de los datos y la modelización de los fenómenos necesarios para el funcionamiento de sitios observatorios en el seno de localidades-talleres en medio tropical.

\section{La última meta, la creación de redes de observatorios de vigilancia eco-epidemiológica}

Es bien conocido que la progresión del conocimiento científico nace fundamentalmente de un mecanismo acumulativo. En el área de las ciencias humanas, los observatorios han demostrado su interés como conjuntos de conocimientos adquiridos, verificados y evaluados, gracias a los cuales la investigación puede desarrollarse en un contínuo permanente (Clignet, 
1998). Los observatorios son instrumentos necesarios para estudiar fenómenos a la vez temporales y espaciales cuya dinámica es más importante que su estática. La vigilancia climática por satélite es quizás el mejor ejemplo de funcionamiento en redes de observación jerarquizadas. Los datos observados alimentan modelos de previsión del tiempo, autorizan el seguimiento de fenómenos y apoyan claramente ensayos de explicación. Los observatorios que analizan las modificaciones del medio ambiente consecuentes a la acción del hombre son tambien órganos de interés para obtener información sobre su dinámica. En el campo de la investigación para la salud, los ejemplos son raros. Existen diferentes sistemas de vigilancia epidemiológica basados fundamentalemente en la detección de casos, una vez estos manifiestan la enfermedad. Es el caso, por ejemplo, de ProMED-mail para la vigilancia global de enfermedades emergentes que reúne más de 20.000 personas en casi 150 países (Woodall, 2001); otro ejemplo es el Instituto de Vigilancia Sanitaria en Francia (Girard, 1995) cuya misión es desarrollar la vigilancia de las enfermedades infecciosas, la declaración obligatoria de los riesgos infecciosos de origen alimentario, de las infecciones nosocomiales, de los riesgos ligados a la polución del aire, a la exposición al amianto o a los poluyentes químicos, a las radiaciones ionisantes, a las enfermedades crónicas o a los traumatismos.

Los métodos utilizados por los observatorios se sitúan fundamentalmente entre la reconstitución retrospectiva del fenoméno y un seguimiento contínuo, presente y futuro, en el espacio y en el tiempo. Por otro lado, la utilización de técnicas informáticas obliga a sistematizar las etapas de la concepción, de la creación y de la puesta en funcionamiento de los observatorios. Los sistemas de información geográficos, programas informatizados que reducen a la misma escala la dinámica de los diferentes parámetros, necesitan una infraestructura bien precisa y la creación de bancos de datos.

La primera estapa consiste entonces en constituir un corpus de datos sobre los ecosistemas naturales y modificados por las actividades humanas y los ciclos naturales de infecciones de origen vírico (arbovirosis) o parasitario (paludismo, leishmaniosis, enfermedad de Chagas). La segunda etapa debe definir los parámetros representativos del fenómeno de estudio y jerarquizarlos según criterios bien establecidos y explicados. La tercera etapa debe permitir el análisis a una escala superior.

Una vez estén estas etapas establecidas, se dispondrá de un útil informatizado, capaz de ser utilizado por operadores y estructuras institucionales. Un sitio internet permitirá poner a disposición de los usuarios los bancos de datos actualizados al mismo tiempo que se podrá disponer de los modelos informatizados para el seguimiento de la dinámica del fenómeno.

La definición de las indicaciones para una evaluación cuantitativa de los riesgos no parece emerger como una prioridad en el medio tropical, salvo en ciertas enfermedades como son la tuberculosis, el paludismo o el sida. La necesidad de desarrollar este tipo de reflexión en las enfermedades transmitidas por insectos vectores como complementaria a las acciones de control para la estimación, caracterización y gestión del riesgo deberán ser prioritarios en los proximos años. Y eso por dos razones.

La primera es que hay que situar la dinámica de las interacciones entre medio ambiente y salud pública en un marco geográfico sistemático (Rogers, 2000) que permita concebir un sistema de análisis y caracterización del riesgo para las poblaciones humanas, destinado no solamente a los responsables regionales de salud, sino a todos aquellos que intervienen en la definición de políticas de gestión medioambiental.

La segunda razón es que hay que asegurar la continuidad de los programas de investigación y de formación de científicos de diferentes disciplinas en programas de estudios comunes, facilitando la cooperación regional. Estos observatorios deben entonces operar a la vez a nivel local, regional e internacional, permitiendo la uniformidad de las actividades individuales o de instituciones formales e informales, la caracterización del objeto de estudio, la lectura sistemática del fenómeno, la coherencia de las medidas cualitativas y cuantitativas, la categorización y la objetivación de las muestras y la interacción entre enfoques de investigación fundamental y aplicada. 


\section{Referencias}

CNRS (Centre National de la Recherche Scientifique), 1998. Les Risques Sanitaires et Leurs Enjeux dans les Démocraties Pluralistes: Perception, Evaluation et Gestion. Quelles Recherches Développer? Paris: CNRS.

CLIGNET, R., 1998. Observatoires du Développement, Observatoires pour le Développement. Paris: Institut Français de Recherche Scientifique pour le Développement en Coopération.

COURA, J. R.; JUNQUEIRA, A. C.; FERNANDES, O.; VALENTE, S. A. \& MILES, M. A., 2002. Emerging Chagas disease in Amazonian Brazil. Trends in Parasitology, 18:171-176.

De La ROCQUE, S.; MICHEL, J. F.; De WISPELAERE, G. \& CUISANCE, D., 2001. New tools for the study of animal trypanosomiasis in the Sudan: Modelbuilding of dangerous epidemiological passage by remote sensing geographic information systems. Parasite, 8:171-195.

DIAS, J. C., 1988. Control of Chagas' disease vectors in Brazil and risk of domiciliary reinfestation by secondary vectors. Memórias do Instituto Oswaldo Cruz, 83:387-391.

DIAS, J. C., 1991. Chagas disease control in Brazil: Which strategy after the attack phase? Annales de la Société Belge de Médicine Tropicale, 71:75-86.

DIAS, J. C. P., 2000. Vigilância epidemiológica em doença de Chagas. Cadernos de Saúde Pública, 16 (Sup. 2):43-59.

DUJARDIN, J. P.; PANZERA, P. \& SCHOFIELD, C. J., 1999. Triatominae as a model of morphological plasticity under ecological pressure. Memórias do Instituto Oswaldo Cruz, 94:223-228.

FUNASA (Fundação Nacional de Saúde)/MS (Ministerio da Saúde), 1998. Gerência de Chagas. Relatorio Técnico. Brasília: MS.

FORATTINI, O. P., 1980. Biogeography, origin and distribution of Triatominae domicile dispersal in Brazil. Revista de Saúde Pública, 14:265-299.

FORATTINI, O. P.; FERREIRA, O. A.; RABELLO, E. X.; BARATA, J. M. \& SANTOS, J. L., 1983. Ecological aspects of South American trypanosomiasis. XVII - Development of Triatominae regional domiciliation in an endemic center of Triatoma sordida. Revista de Saúde Pública, 17:159-199.

GARRETT, L., 1994. The Coming Plague. New York: Farrar, Strauss \& Giroux.

GIRARD, J. F., 1995. Sentinel networks. The national public health network. State information. Bulletin de l'Académie Nationale de Médecine, 179:919925.

HECKADON-MORENO, S.; IBANEZ, R. \& CONDIT, R., 1999. La Cuenca del Canal: Deforestación, contaminación y urbanización. Proyecto de Monitoreo de la Cuenca del Canal de Panamá. Ciudad de Panama: Instituto Smithsonian de Investigaciones Tropicales.

HOARE, C. A., 1962. Reservoir hosts and natural foci of human protozoal infections. Acta Tropica, 19: 281-317.

MMA (Ministério do Meio Ambiente), 1998. Primeiro Relatório Nacional para a Convenção sobre Diversidade Biológico - Brasil. Brasília: MMA.

MT (Ministério dos Transportes), 2003. Avança Brasil
2000-2003 - Brasil Integrado. 23 Janeiro 2003 $<$ http://www.transportes.gov.br/bit/avancabr/avbrpp.html>.

NEPSTAD, D.; VERISSIMO, A.; MOUTINHO, P. \& NOBRE, C., 2000. O empobrecimento oculto da floresta amazônica. Ciência Hoje, 27:70-73.

PAVLOVSKY, E. N., 1939. Natural Nidality of Transmissible Diseases in Relation to Lanscape. Epidemiology of Zooanthropozoonoses. Moscow: Peace Publications.

PETRISCHEVA, P. A., 1971. Natural focality of leishmaniasis in the U.R.S.S. Bulletin of the World Health Organization, 44:567-570.

RIOUX, J. A., 1977. Ecologie d'un foyer méditérranéen de leishmaniose viscérale. Essai de modélisation. In: Ecologie des Leishmanioses (Centre National de la Recherche Scientifique - CNRS, ed.), pp. 295-304, Montpellier: CNRS.

RIOUX, J. A., 2001. Trente ans de coopération francomarocaine sur les leishmanioses: Dépistage et analyse des foyers. Facteurs de risque. Changements climatiques et dynamique noso-géographique. Revue de l'Association des Anciens Elèves de l'Institut Pasteur, 168:90-100.

RIOUX, J. A.; DEREURE, J. \& PERIERES, J., 1990. Approche écologique du risque épidémiologique. L'exemple des leishmanioses. Bulletin d'Ecologie, 21:1-9.

RIOUX, J. A.; GOLVAN, Y. J.; CROSET, H.; TOUR, S.; HOUIN, R.; ABONNENC, E.; PETIDIDIER, M.; VOLLHARDT, Y.; DEDET, J. P.; ALBARET, J. L.; LANOTTE, G. \& QUILICI, M., 1969. Epidémiologie des Leishmanioses dans le Sud de la France. Paris: Institut National de la Santé et de la Recherche Médicale.

ROGERS, D. J., 2000. Satellites, space, time and the African trypanosomiases. Advances in Parasitology, 47:129-171.

ROMAÑA, C. A.; PIZARRO, J. C.; RODAS, E. \& GUILBERT, E., 1999. Palm trees as ecological indicators of risk areas for Chagas disease. Transactions of the Royal Society of Tropical Medecine and Hygiene, 93:1-2.

SCHATZMAYER, H. G., 2000. Dengue situation in Brazil by year 2000. Memórias do Instituto Oswaldo Cruz, 95:179-181.

SCHOFIELD, C. J.; DIOTAIUTI, L. G. \& DUJARDIN, J.-P., 1999. The process of domestication in Triatominae. Memórias do Instituto Oswaldo Cruz, 94:375-378.

SILVA, L. J., 1999. A Evolução da Doença de Chagas no Estado de São Paulo. São Paulo: Editora Hucitec.

SILVEIRA, A. C. \& VINHAES, M., 1999. Elimination of vector-borne transmission of Chagas disease. Memórias do Instituto Oswaldo Cruz, 94(Sup. 1): 405-411.

SORRE, M., 1943. Les Fondements Biologiques de la Géographie Humaine. Paris: Colin.

WALKER, D. H.; BARBOUR, A. G.; OLIVER, J. H.; LANE, R. S.; DUMLER, J. S.; DENNIS, D. T.; PERSING, D. H.; AZAD, A. F. \& McSWEEGAN, E., 1996. Emerging bacterial zoonotic and vector-borne diseases. Ecological and epidemiological factors. JAMA, 275:463-469. 
WILSON, M. E.; LEVINS, R. \& SPIELMAN, A., 1994.

Disease in evolution: Global changes and emergence of infectious diseases. Annals of the New York Academy of Sciences, 70:740-747.

WOODALL, J. P., 2001. Global surveillance of emerging diseases: The ProMED-mail perspective. Cadernos de Saúde Pública, 17(Sup.):147-154.

ZELEDON, R. \& RABINOVICH, J. E., 1981. Chagas' disease: An ecological appraisal with special emphasis on its insects vectors. Annals of the Revue of Entomology, 26:101-133.

Recibido el 18 de mayo de 2000

Versión final presentada el 15 de octubre de 2000

Aprobado el 12 de diciembre de 2000 